\title{
The role of mirroring and mentalizing networks in mediating action intentions in autism
}

\author{
Lauren E Libero', Jose O Maximo ${ }^{1}$, Hrishikesh D Deshpande², Laura G Klinger ${ }^{3}$, Mark R Klinger ${ }^{4}$ \\ and Rajesh K Kana ${ }^{1 *}$
}

\begin{abstract}
Background: The ability to interpret agents' intent from their actions is a vital skill in successful social interaction. However, individuals with autism spectrum disorders (ASD) have been found to have difficulty in attributing intentions to others. The present study investigated the neural mechanisms of inferring intentions from actions in individuals with ASD.

Methods: Functional magnetic resonance imaging (fMRI) data were acquired from 21 high-functioning young adults with ASD and 22 typically developing (TD) control participants, while making judgments about the means (how an action is performed) and intention (why an action is performed) of a model's actions.

Results: Across both groups of participants, the middle and superior temporal cortex, extending to temporoparietal junction, and posterior cingulate cortex, responded significantly to inferring the intent of an action, while inferior parietal lobule and occipital cortices were active for judgments about the means of an action. Participants with ASD had significantly reduced activation in calcarine sulcus and significantly increased activation in left inferior frontal gyrus, compared to TD peers, while attending to the intentions of actions. Also, ASD participants had weaker functional connectivity between frontal and posterior temporal regions while processing intentions.

Conclusions: These results suggest that processing actions and intentions may not be mutually exclusive, with reliance on mirroring and mentalizing mechanisms mediating action understanding. Overall, inferring information about others' actions involves activation of the mirror neuron system and theory-of-mind regions, and this activation (and the synchrony between activated brain regions) appears altered in young adults with ASD.
\end{abstract}

Keywords: Action, Intention, Means, fMRI, Autism spectrum disorders, Mirror neuron system, Theory-of-mind

\section{Background}

As social animals, human beings are often engaged in watching and interpreting each other's behavior, especially the 'how', 'what', and 'why' of their actions [1]. A basic understanding of the nature and means of an action helps observers infer the intention of the agent involved. Simulating others' actions may prove critical in helping us understand the meaning of such actions and emotions, as suggested by earlier studies of the simulation theory of mindreading [2-4]. However, more recently it has been suggested that, at the neural level, the representation of one's own and others' actions is mediated by the mirror

\footnotetext{
* Correspondence: rkana@uab.edu

'Department of Psychology, University of Alabama at Birmingham, CIRC

235G, 1719 6th Ave South, Birmingham, AL 35294-0021, USA

Full list of author information is available at the end of the article
}

neuron system (MNS) through internal simulation and replication of these actions [2,5-7]. First discovered in the macaque monkey, mirror neurons are a set of specific neurons that fire when the monkey executes a movement, as well as when it observes the same movement performed by someone else [8-12], creating a simulation circuit that allows the association of one's own actions with the actions of others. Human neuroimaging studies have pointed to the ventral premotor cortex, especially the inferior frontal gyrus (IFG), along with the inferior parietal lobule (IPL), as the core regions of mirror neuron activity $[6,7,9,13-15]$. Nonetheless, questions remain about the significance of the MNS and its putative involvement in making social inferences. For example, how does one understand the meaning and intent of others' actions by simulating it? Is simulation, mediated by MNS activity, 
necessary and sufficient for understanding an agent's action intention?

It is assumed that the information about a simulated action in the MNS is passed along to the core theory-ofmind (ToM - the ability to attribute mental states to others) regions for inferring an agent's intention [16-18]. Areas found to be associated with ToM include the medial prefrontal cortex (MPFC), the posterior cingulate cortex (PCC), and the posterior superior temporal sulcus (pSTS) at the temporoparietal junction (TPJ) [19-23]. Both MNS and ToM networks are involved in understanding actions and attributing intentions, however they appear to be functionally and anatomically segregated, at least in some ways. One meta-analysis of over 200 functional magnetic resonance imaging (fMRI) activation studies showed that the MNS network (IPL and IFG) was related to processing biological motion (moving body parts) and ToM network (MPFC, PCC, and TPJ) to more abstract processing of others' intentions, in the absence of any biological motion [24]. In addition, two cross-sectional fMRI studies $[18,25]$ specifically explored the how, what, and why of others' actions in typically developing (TD) individuals. Both studies concluded that the MNS (through the IFG aspect) is active during the visual processing of others' actions (how and what), and ToM regions (MPFC, PCC, and PSTS) are additionally recruited to process their intentions (why); thus it appears that inferring others' intentions from their actions may be the result of two distinct functions (MNS and ToM networks).

While understanding the means of actions (how) and attributing intentions to the agent (why) may be an implicit and rather effortless task for TD individuals, several behavioral studies have found that people with autism spectrum disorders (ASD) have great difficulty in doing so [26-29]. One early study [30] suggested that an interruption in the ability to connect one's own actions with the actions of others results in impaired social development and a deficit in ToM. Behavioral studies have indicated that individuals with ASD have a marked difficulty in connecting another's actions to their own, particularly due to impaired imitation skills, a behavioral correlate of MNS [29,31-33]. Since the ability to imitate may strongly rely on MNS activity [34-38], it is not surprising that a dysfunction in the MNS has been postulated as an underlying mechanism for imitation deficits seen in ASD $[29,39]$. Studies have linked the MNS with other aspects of social cognition, including empathy, joint attention, self-recognition $[29,36,40]$, and ToM [41]. In addition, anatomical and functional alterations of the MNS have been found to be related to social impairment and symptom severity in ASD [29,39,42-46] (for a review see [47]). Therefore, a dysfunctional MNS may underlie the widespread social impairments seen in ASD (see reviews by [48-50]).
Despite the findings of dysfunctional MNS, other studies have reported no differences in MNS activation in ASD participants compared to TD individuals, raising questions about a MNS dysfunction in ASD [51-53]. These critiques allude to the possibility of different neurological processes occurring at resting state in autism, triggering false detection of hypoactivation in MNS [52], the possible existence of multiple mirroring systems in autism [54,55], and even methodological differences in fMRI studies [56]. Even the proponents of the MNS dysfunction hypothesis of ASD acknowledge that a deficit in the MNS, although able to explain some social-cognitive dysfunctions, as of yet does not explain other key symptoms of ASD, such as repetitive behaviors and restricted interests [39]. Therefore, the MNS hypothesis of ASD is a topic of intense debate in the autism literature.

The present study addresses several questions pertaining to the MNS hypothesis, the involvement of MNS and ToM networks in action understanding and mentalizing, and how these networks may be functionally different in people with ASD. Using a set of action intention stimuli adapted from de Lange et al. [18], we predicted a significant increase in MNS (IFG and IPL) response while attending to the means (how) of an action, whereas increased recruitment of ToM (MPFC, PCC, and TPJ) regions when attending to the intent (why) behind an action in TD individuals. Given the somewhat inconsistent literature in MNS in ASD, but consistent findings of abnormal ToM processing in ASD [57-59], we expect abnormal recruitment of the ToM network while attending to intentions, but rather typical MNS activation while attending to the means of an action in ASD participants. Based on the disrupted connectivity accounts of ASD [60-63], it is predicted that the ASD participants will exhibit altered functional connectivity (synchronization of brain activity) of the MNS and ToM networks during this task. The current study is novel in its focus on examining the differential involvement of MNS and ToM networks and their functional connectivity in autism. Findings from this study will provide new insights into understanding the role of MNS and ToM systems in action understanding and its potential alterations in ASD.

\section{Methods}

\section{Participants}

Functional MRI data were collected from 26 highfunctioning young adults with ASD and 28 TD control participants. Five ASD and 6 TD participants with excessive head motion were excluded from the analysis. Groups were matched on age, handedness, IQ, and head motion, resulting in a final sample of 22 TD and 21 ASD participants (see Table 1). All participants were assessed on the 
Table 1 Demographic information

\begin{tabular}{|c|c|c|c|c|}
\hline & \multicolumn{2}{|l|}{ Groups } & \multirow[b]{2}{*}{$t$ value } & \multirow[b]{2}{*}{$p$ value } \\
\hline & $T D(n=22)$ & ASD $(n=21)$ & & \\
\hline Gender & $17 \mathrm{M} ; 5 \mathrm{~F}$ & $17 \mathrm{M} ; 4 \mathrm{~F}$ & - & - \\
\hline Handedness & $20 \mathrm{R}, 1 \mathrm{~L}, 1 \mathrm{~A}$ & $16 \mathrm{R}, 2 \mathrm{~L}, 3 \mathrm{~A}$ & - & - \\
\hline Age (years) & $24.9( \pm 5.2 ; 19-36)$ & $25.7( \pm 6.4 ;$ 17-40) & 0.43 & 0.67 \\
\hline Verbal IQ & $114.4( \pm 10.3 ; 92-141)$ & $112.9( \pm 15.0 ; 80-139)$ & 0.37 & 0.71 \\
\hline Performance IQ & $116.5( \pm 9.5 ; 100-133)$ & $106.3( \pm 12.4 ; 94-138)$ & 0.05 & 0.96 \\
\hline Full-scale IQ & $117.5( \pm 8.8 ; 105-140)$ & $116.3( \pm 12.8 ; 91-140)$ & 0.35 & 0.73 \\
\hline RAADS-R total & $45.7( \pm 24.1 ; 13-112)$ & $115.4( \pm 39.5 ; 32-181)$ & 3.54 & $<0.001$ \\
\hline Mind in the Eyes & $21( \pm 2.3 ; 17-25)$ & $19( \pm 3.5 ; 15-25)$ & 2.25 & 0.03 \\
\hline EQ & $47.5( \pm 10.3 ; 27-74)$ & $31.5( \pm 13.3 ; 15-65)$ & 3.54 & $<0.001$ \\
\hline
\end{tabular}

Values are presented as mean (standard deviation; range). The $P$ value is independent $t$-tests for differences between groups. Abbreviations: TD typically developing, $A S D$ autism spectrum disorder, $A$ ambidextrous, $F$ female, $L$ left, $M$ male, $R$ right, $I Q$ intelligence quotient, $R A A D S-R$ Ritvo Autism Asperger Diagnostic Scale-Revised, EQ Empathy Quotient. EQ scores missing for one ASD participant due to incomplete questionnaire. Mind in the Eyes scores missing for one TD participant who did not complete the test.

Wechsler Abbreviated Scale of Intelligence (WASI; [64]), Edinburgh Handedness Inventory [65], Reading the Mind in the Eyes Test ([66]), the Ritvo Autism Asperger Diagnostic Scale-Revised (RAADS-R; [67]), and the Empathy Quotient (EQ; [68]). All participants were required to have a full-scale IQ of 80 or above (measured by the WASI) to be included in this study. The participants with ASD were recruited through service providers in Alabama (the University of Alabama at Birmingham (UAB) Civitan-Sparks Clinic, UA Autism Spectrum Disorders Clinic, Glenwood Foundation, Mitchell's Place, and the Alabama Autism Society), and from a roster of individuals who have participated in previous studies from the Cognition, Brain, and Autism Lab at UAB. Participants with ASD were included if they had a diagnosis of ASD based on the Autism Diagnostic Interview (ADI-R; [69]) and the Autism Diagnostic Observation Schedule (ADOS; [70]). Diagnoses were verified through patient records retrieved through each participant's clinician. Healthy controls were recruited through advertisements placed in the Birmingham area, and through the Introduction to Psychology course (PY 101) subject pool of the UAB Department of Psychology. Participants were excluded from the study if they reported any neurological disorders, claustrophobia, a body mass index exceeding 34, metal implants or a history of working with metal, kidney disease, diabetes, hypertension, anemia, sickle cell disease, or if they were taking psychotropic medications. Before participating in the study, study procedures were fully explained to all participants and informed consent for all aspects of the study protocol was obtained for all participants. All aspects of the study protocol and consent form were approved by the ethics committee of the UAB Institutional Review Board for Human Use (Protocol \#: F070824010, Assurance \#: FWA00005960).

\section{Stimuli and experimental design}

The stimuli, adapted from de Lange et al. [18] included a series of static, color images of a model interacting with various common household objects (see Figure 1). The objects included were small, handheld items such as a telephone, coffee cup, spoon, or camera. The stimuli were presented in a blocked design format with two tasks (determining intentions and judging the means of actions) and a fixation condition that served as a baseline. Each image, presented for four seconds during the experiment, was unique, and presentations of the blocks were randomized and counterbalanced across participants. In the intention task, participants viewed an image and determined whether the intention behind the model's action was ordinary or unusual

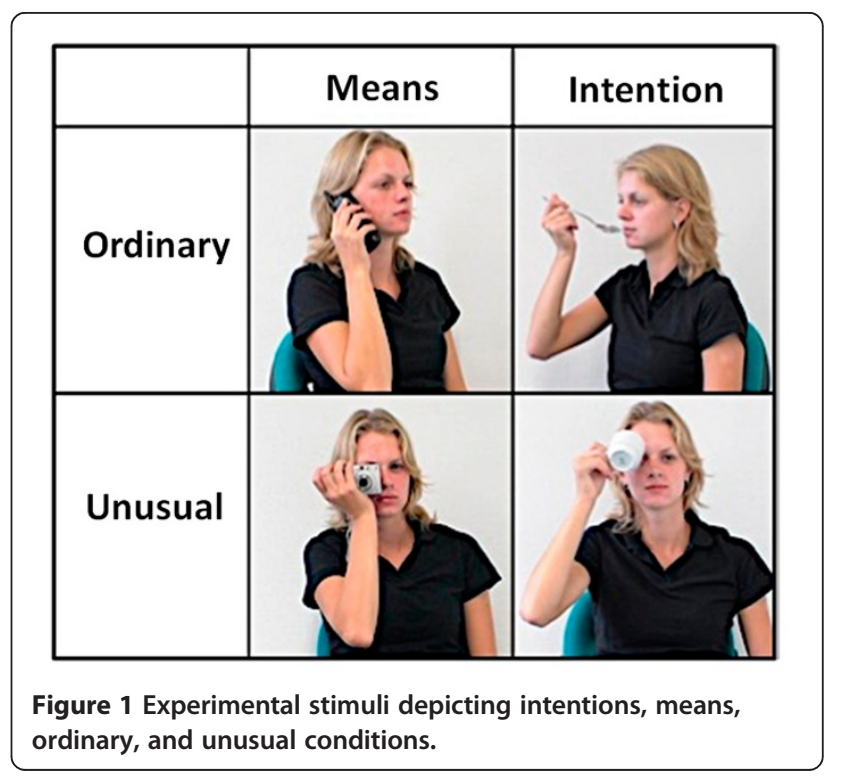


(but always presented one category in a given block). In the means task, participants viewed an image and determined whether the means (or the way in which the action was carried out) of the model's action was ordinary or unusual. Brief instructions for the tasks were presented at the beginning of each block, and participants indicated their response (ordinary or unusual) via button press. Each block consisted of four pictures with an inter-stimulus interval of one second. The experiment was comprised of 16 blocks, with eight blocks for the intention task and eight blocks for the means task. In total, 32 images per condition were included, with a total of 64 images for a complete run. Each block contained either ordinary or unusual trials, but never ordinary and unusual, to facilitate the comparison of these conditions in addition to intention and means.

Each participant practiced the experiment on a laptop computer before the scanning session. The practice included a fixation trial, as well as several unique trials (using images from the same stimuli set that were not included in the fMRI trials) representative of the intention and means conditions. While in the MRI scanner, the software Inquisit 2 (Millisecond Software, Seattle, United States) was used to visually present the stimuli. Using a laptop computer and an LCD projector, the stimuli were projected onto a screen behind the participant's head, which were viewed using a mirror. The participants' responses were recorded using fiber optic buttons, with unusual corresponding to the right index finger, and ordinary to the right middle finger. The participant responses provided reaction time and performance accuracy data. Behavioral data were compared between groups using a two (group) $\times$ two (condition) mixed analysis of variance (ANOVA).

\section{MRI data acquisition}

Imaging data were acquired using a $3 \mathrm{~T}$ Siemens Allegra scanner (Siemens Medical Inc., Erlangen, Germany) housed at the Civitan International Research Center, UAB. Anatomical images were acquired using high resolution T1-weighted scans using a 160-slice three-dimensional magnetization-prepared rapid gradient-echo (MPRAGE) volume scan (Repetition time $[\mathrm{TR}]=200 \mathrm{~ms}$; echo time $[\mathrm{TE}]=3.34 \mathrm{~ms}$; flip angle $=12^{\circ}$; field of view $[$ FOV $]=25.6 \mathrm{~cm}$; matrix size $=256 \times 256$; slice thickness $=1 \mathrm{~mm})$. Functional $\mathrm{T} 2 *$-weighted images $(\mathrm{T} 2 *$ is a time constant describing the exponential decay of MRI signal) were obtained using a single-shot gradientrecalled echo-planar pulse sequence $(\mathrm{TR}=1000 \mathrm{~ms}$, $\mathrm{TE}=30 \mathrm{~ms}$, flip angle $=60^{\circ}, \mathrm{FOV}=24 \mathrm{~cm}$, matrix $=$ $64 \times 64$. This sequence allowed rapid image acquisition and covered most of the brain (17 5-mm thick slices with a 1-mm gap in an oblique-axial orientation) in a single cycle of scanning with an in-plane resolution of $3.75 \times$ $3.75 \times 5 \mathrm{~mm}^{3}$. A TR of one second was used in order to elicit better temporal resolution for the functional connectivity analyses. Relatively thicker slices were used to allow for higher signal-to-noise ratio.

\section{Data preprocessing}

Functional images were processed using a combination of Analysis of Functional NeuroImages software (NIMH Scientific and Statistical Computing Core, Bethesda, MD, USA) (AFNI, [71]) and FMRI software library 5.0 (Analysis Group, FMRIB, Oxford, UK) (FSL, [72]). Functional images were slice-time corrected, and correction for head motion was performed by registering each functional volume to the middle time point of the scan. These images were then registered to the anatomical images via FSL's FMRIB's Linear Image Registration Tool (FLIRT) [73,74]. Both images were resampled (3 $\mathrm{mm}$ isotropic) and standardized to the atlas space of the MNI152 template via FSL's nonlinear registration tool (FNIRT) for group comparisons, and a global full width at half maximum of $8 \mathrm{~mm}$ was applied.

\section{fMRI activation analysis}

Functional images were scaled to a mean of 100 and whole-brain statistical analyses were performed on an individual basis using a standard general linear model approach via AFNI's 3dDeconvolve function with ordinary intention, ordinary means, unusual intention, and unusual means trials as regressors of interest. Six rigid-body motion parameters acquired from head motion correction were treated as covariates. The following two orthogonal contrasts were computed to assess average brain response: intention (ordinary + unusual) versus means (ordinary + unusual), and unusual (intention + means) versus ordinary (intention + means). Areas of statistically significant activation were determined using one- and twosample $t$-tests, and to correct for multiple comparisons, 10,000 Monte Carlo simulations were applied via AFNI's 3dClustSim function to obtain a corrected significance level of $P<0.05$.

We further examined the relationship between fMRI blood-oxygen-level dependent (BOLD) activation driven by the tasks with neuropsychological assessment data by conducting exploratory correlational analysis between beta coefficients from the contrast intention versus means with EQ and RAADS-R total scores. The regions of interest (ROI) for this analysis were defined on the group activation map for the pooled sample (TD + ASD) using the contrast intention versus means, (peak positive and negative $t$-values) so that it best represents the ROIs for both conditions. A total of 12 ROI's relevant to these tasks were identified. For intention: left and right middle frontal gyrus (LMFG and RMFG), left and right superior 
temporal sulcus (LSTS and RSTS), left and right temporoparietal junction (LTPJ and RTPJ), and posterior cingulate cortex (PCC). For means: middle cingulate (MCIN), left and right inferior parietal lobule (LIPL and RIPL), and left and right extrastriate body area (LEBA and REBA). Seeds were created using spherical binary masks $(8-\mathrm{mm}$ and $12-\mathrm{mm}$ radius) that best captured the cluster of activation and were trimmed from white matter. Parameter estimates were then extracted from this contrast on an individual basis on each group. We assessed brain-behavior relationships separately for the TD, and ASD groups, and for both groups combined.

\section{Functional connectivity analysis}

First, to account for signal from cerebral white matter and lateral ventricles, masks were created at the participant level using FSL's FMRIB's Automated Segmentation Tool (FAST) automated segmentation [75]. Masks were trimmed to avoid partial-volume effects, and an average time series for each region was extracted. Derivatives for motion parameters, white matter, and ventricular time series were computed. Following spatial smoothing, sources of noise (head motion, white matter, and lateral ventricles plus derivatives) were modeled and removed using a general linear model, and residual time series were used in subsequent functional connectivity analysis. These steps characterize this analysis as co-activation functional connectivity MRI (fcMRI) and since there is no low-pass filtering or task regression, this analysis retains task-driven effects [76]. Then, residual time series were segmented into the two experimental conditions of interest (intention and means) in order to assess connectivity between the tasks. The ROIs for this analysis were the same as the ones described in the earlier paragraph. Residual time series were extracted for each seed, and correlation coefficients were calculated across the residual time courses from other ROIs. Correlation coefficients were $z$-transformed using an inverse hyperbolic tangent function, followed by direct comparison of the $z$-transformed correlations between the TD and ASD groups using two-sample $t$-tests. Given the large number of comparisons, increasing the likelihood of type I error, a follow-up connectivity network analysis was performed by grouping the ROIs into networks based on their anatomical locations in the brain. Four networks were created for this analysis: frontal (LMFG and RMFG), temporal (LTPJ, RTPJ, LSTS, and RSTS), posterior (LIPL, RIPL, LEBA, and REBA), and medial (PCC and MCIN), and statistical analyses were performed using the same procedure as described above.

\section{Head motion correction}

Because head motion can impact functional connectivity analyses $[77,78]$, the following precautions were taken. Head motion was quantified as the Euclidean distance calculated from the six rigid-body motion parameters for two consecutive time points. Any instance under $0.5 \mathrm{~mm}$ was considered to be excessive motion, and the time point as well as the immediately preceding and subsequent time points were censored, or scrubbed [79]. If two censored time points occurred within 10 time points of each other, all time points between them were also censored. All participants retained $80 \%$ of time points after censoring. Average head motion over each participant's session was defined as the root mean square of displacement and did not significantly differ between groups $(t(41)=-1.49, P=0.14)$. For more detailed analysis of head motion, a two-way ANOVA was conducted to test the effects of group (TD and ASD) and type of motion (three translational and three rotational). The interaction of group and motion type was not significant (F-value $(\mathrm{F})(5,276)=1.11, P=0.41)$.

\section{Results}

\section{Overview}

The main results of this study are as follows: 1) processing intentions activated middle and superior temporal cortex along with PCC and precuneus (part of the ToM network) in both TD and ASD groups, whereas processing means activated IPL (part of the MNS network) and occipital areas; 2) processing both ordinary and unusual actions showed strong activation in both groups in frontal, middle temporal, and parietal areas; 3) group differences involved significantly reduced activation in ASD, relative to TD, in calcarine sulcus extending to PCC during intention, and increased activation in the ASD group in LIFG while processing means of an action; 4) the ASD group showed increased activation in pre- and postcentral gyrus, RIFG, and LIPL with no inverse effects found (TD > ASD) during unusual actions; 5) weaker functional connectivity was found in the ASD group between frontal and posterior temporal regions while processing action intentions; 6) brain-behavior correlations were also seen in terms of empathizing ability (positive) and autism symptom severity (negative) related to activation in PCC; and 7) a significant difference in performance accuracy, but not in reaction time, was also found between ASD and TD groups.

\section{Behavioral data}

To assess possible group differences in performance accuracy and reaction time (RT) measured during the fMRI task, we conducted a two Group (ASD versus TD) $\times$ two condition (means versus intentions) mixed ANOVA. Group was treated as a between-subject factor and intention and means as within-subject variables. There was a significant main effect of group in performance accuracy (ASD means trials: mean $(\mathrm{M})=83 \%$, standard deviation $(\mathrm{SD})=12 \%$; ASD intention trials: $\mathrm{M}=84 \%$, $\mathrm{SD}=12 \%$; $\mathrm{TD}$ means trials: $\mathrm{M}=92 \%, \mathrm{SD}=9 \%$; 
TD intention trials: $\mathrm{M}=89 \%, \mathrm{SD}=12 \% ; \mathrm{F}(2,41)=5.40$, $P=0.03)$. However, there was no significant main effect of condition $(\mathrm{F}(2,41)=0.61, P=0.44)$, and no significant interaction between group and condition for accuracy $(\mathrm{F}(2,41)=1.56, P=0.22)$. For reaction time, there was no significant main effect of group (ASD means trials: $\mathrm{M}=$ 1,903 ms, SD = 504; ASD intention trials: $M=1,791 \mathrm{~ms}$, $\mathrm{SD}=431$; TD means trials: $\mathrm{M}=1702 \mathrm{~ms}, \mathrm{SD}=436$; $\mathrm{TD}$ intention trials: $\mathrm{M}=1684 \mathrm{~ms}, \mathrm{SD}=572), \mathrm{F}(2,41)=0.99$, $P=0.33)$. There was also no significant main effect of condition $(\mathrm{F}(2,41)=1.98, P=0.17)$, nor was there a significant interaction between group and condition for reaction time $(\mathrm{F}(2,41)=1.05, P=0.31)$.

\section{Brain activation}

\section{Within-group results}

We hypothesized the involvement of MNS and ToM networks in this task of action understanding, with differential roles in detecting the means and intention. Processing intention in contrast to means revealed robust patterns of activation in both groups in regions associated with ToM, including middle and superior temporal cortex extending to TPJ, and PCC. The means of executing an action, on the other hand, showed significant activation in bilateral IPL, and left middle/inferior occipital areas (see Figure 2A, B and Table 2). Thus, while detecting intentions recruited regions associated with the ToM network, processing means evoked activation in MNS areas like IPL. In addition, in the contrast unusual versus ordinary, activation was detected in the bilateral IPL, bilateral extrastriate body area (EBA), left IFG, and left supplementary motor area (SMA) for both groups; whereas, ordinary actions (relative to unusual actions) showed activation in postcentral gyrus and calcarine sulcus in both groups (all significant clusters at $P<0.05$, corrected; see Figure $3 \mathrm{~A}, \mathrm{~B}$ and Table 3 ). There were two significant correlations between activation (for the contrast intention versus means) in the PCC with EQ, and RAADS-R total in the pooled sample (TD + ASD). The relationship between PCC and EQ was positive (correlation coefficient $(r)=0.33$, $P=0.02$ ), whereas a negative relationship was found with the RAADS-R total $(r=-0.35, P=0.01)$. However, these results should be interpreted with caution due to two reasons: no significant correlations were found when the groups were examined separately, and these correlations did not survive correction for multiple comparisons.

\section{Between-group comparison results}

Between-group comparison analyses for each of the contrasts revealed significant differences between the TD and ASD groups. For the intention versus means contrast, the TD group showed increased activation in the right calcarine sulcus (extending to the PCC), whereas the ASD group showed increased activation in the left IFG. In the unusual versus ordinary contrast, the ASD group showed increased activation in middle orbital gyrus, pre- and postcentral gyrus, and right IPL. No inverse effects (TD > ASD) were found on this contrast (Figures $2 \mathrm{C}$ and $3 \mathrm{C}$ ). Thus the

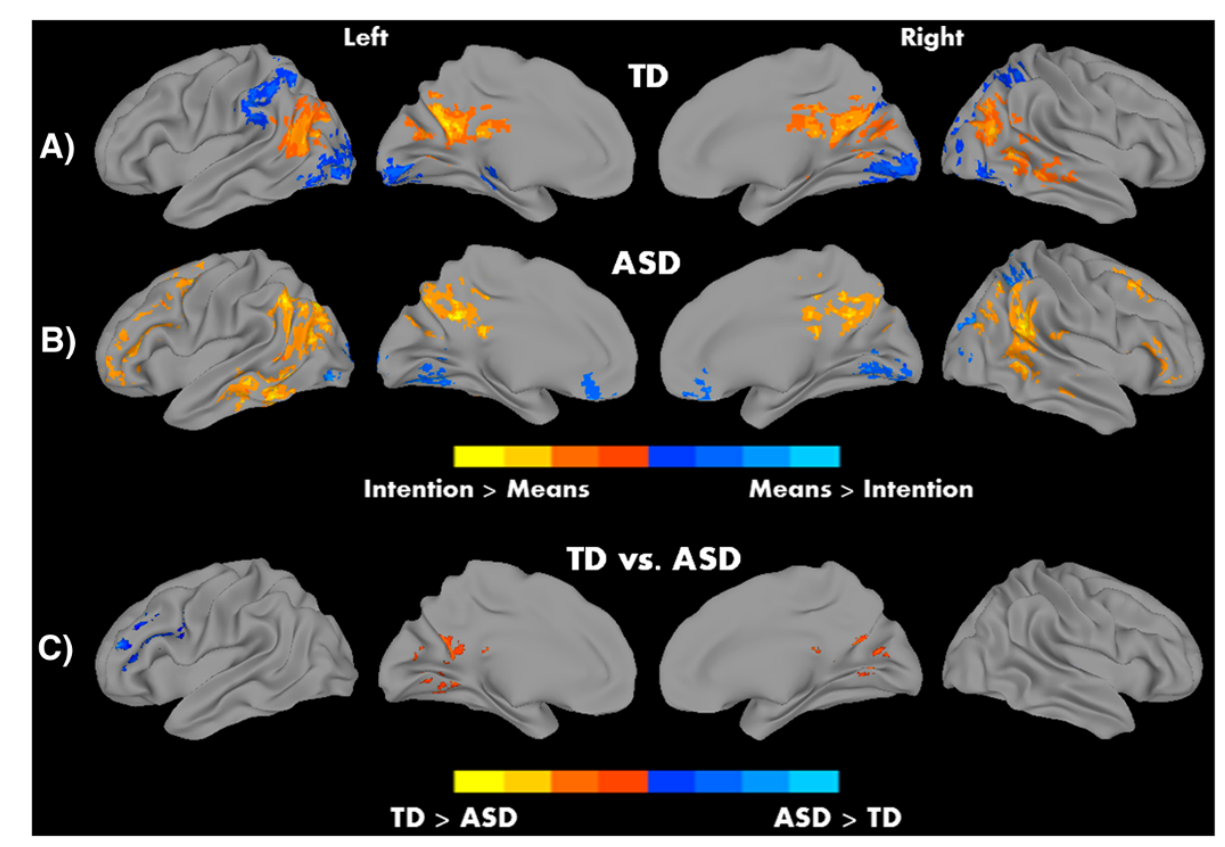

Figure 2 Within-group results for A) typically developing (TD) and B) autism spectrum disorder (ASD) groups, and C) significant between-group differences for the contrast intention versus means. Warm colors represent intention $>$ means and cool colors means $>$ intention for within-group results; $P<0.05$, corrected. 
Table 2 Areas of peak activation for intention versus means and means versus intention contrasts

\begin{tabular}{|c|c|c|c|c|c|c|c|}
\hline \multirow[b]{2}{*}{ Contrast and group } & \multirow[b]{2}{*}{ Region } & \multirow[b]{2}{*}{ Hemisphere } & \multirow{2}{*}{$\begin{array}{l}\text { Volume } \\
\text { (number of voxels) }\end{array}$} & \multicolumn{3}{|c|}{ Peak coordinates } & \multirow{2}{*}{$\begin{array}{l}\text { Peak } \\
t\end{array}$} \\
\hline & & & & $x$ & $Y$ & $\mathrm{Z}$ & \\
\hline \multicolumn{8}{|c|}{ Intention versus means } \\
\hline \multirow[t]{3}{*}{ TD } & Precuneus & Left & 1,063 & -6 & -63 & 33 & 7.4 \\
\hline & Middle temporal gyrus & Right & 616 & 39 & -51 & 3 & 6.8 \\
\hline & Middle temporal gyrus & Left & 482 & -60 & -63 & 18 & 5.9 \\
\hline \multirow[t]{6}{*}{ ASD } & Middle occipital gyrus & Left & 1,056 & -36 & -72 & 36 & 5.2 \\
\hline & Posterior cingulate cortex & Left & 649 & -6 & -51 & 30 & 5.1 \\
\hline & Superior temporal gyrus & Right & 605 & 54 & -45 & 21 & 5.1 \\
\hline & Middle frontal gyrus & Left & 501 & -30 & 3 & 51 & 5.2 \\
\hline & Inferior frontal gyrus & Right & 102 & 57 & 21 & 6 & 3.1 \\
\hline & Middle frontal gyrus & Right & 100 & 36 & 15 & 48 & 3.5 \\
\hline \multicolumn{8}{|c|}{ Means versus intention } \\
\hline \multirow[t]{3}{*}{ TD } & Middle occipital gyrus & Left & 1,564 & -33 & -87 & -3 & -5.3 \\
\hline & Supramarginal gyrus & Left & 334 & -51 & -30 & 33 & -4.0 \\
\hline & Thalamus & Left & 143 & -24 & -27 & -3 & -4.7 \\
\hline \multirow[t]{4}{*}{ ASD } & Cerebellar vermis & Left & 334 & 3 & -57 & -9 & -3.8 \\
\hline & Inferior occipital gyrus & Left & 253 & -27 & -78 & -6 & -4.6 \\
\hline & Angular gyrus & Right & 127 & 30 & -60 & 48 & -4.6 \\
\hline & Rectal gyrus & Left & 108 & 0 & 33 & -24 & -3.3 \\
\hline \multicolumn{8}{|l|}{ Group differences } \\
\hline $\mathrm{TD}>\mathrm{ASD}$ & Calcarine gyrus & Right & 355 & 6 & -66 & 15 & 3.8 \\
\hline$A S D>T D$ & Inferior frontal gyrus & Left & 178 & -48 & 33 & 15 & -3.4 \\
\hline
\end{tabular}

Abbreviations: $T D$ typically developing, $A S D$ autism spectrum disorder.

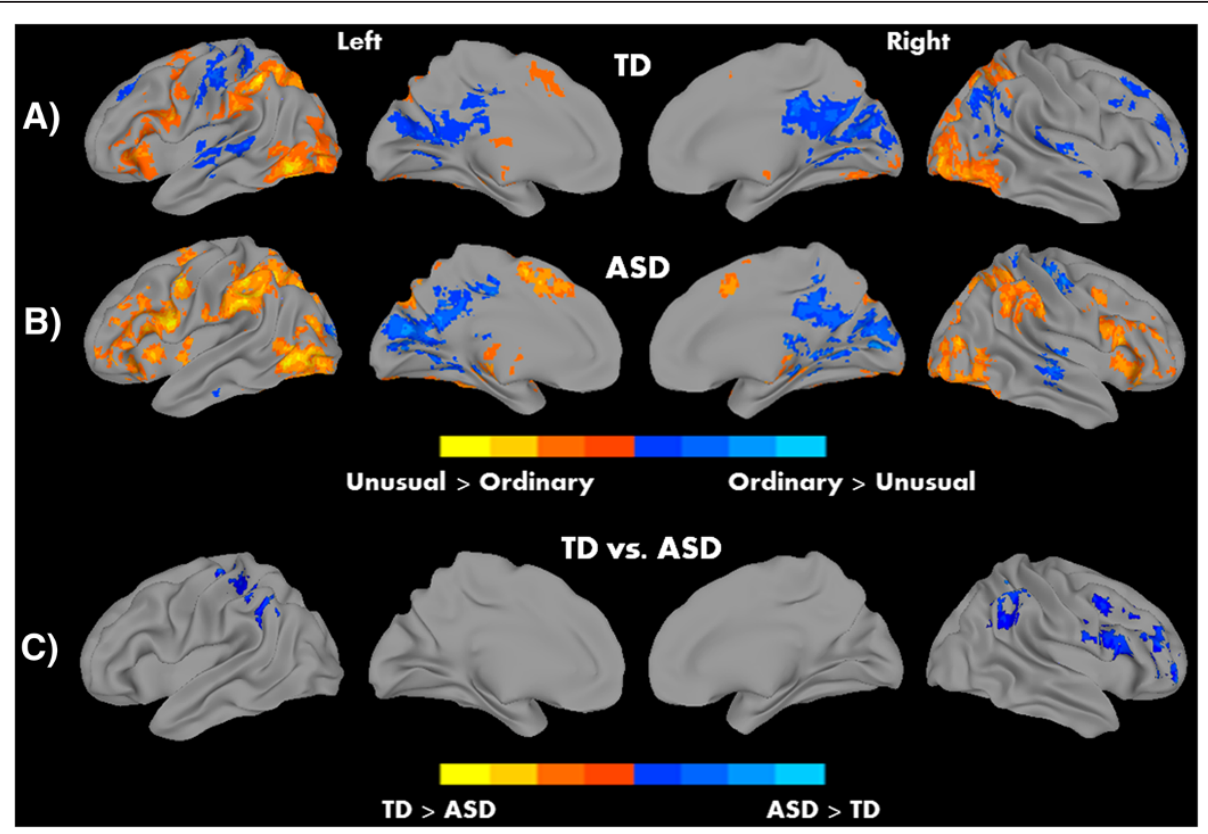

Figure 3 Within-group results for A) typically developing (TD) and B) autism spectrum disorder (ASD) groups, and C) significant between-group differences for the contrast unusual versus ordinary. Warm colors represent Unusual $>$ Ordinary and cool colors Ordinary $>$ Unusual; $P<0.05$, corrected. 
Table 3 Areas of peak activation for unusual versus ordinary and ordinary versus unusual contrasts

\begin{tabular}{|c|c|c|c|c|c|c|c|}
\hline \multirow[b]{2}{*}{ Contrast and group } & \multirow[b]{2}{*}{ Region } & \multirow[b]{2}{*}{ Hemisphere } & \multirow{2}{*}{$\begin{array}{l}\text { Volume } \\
\text { (number of voxels) }\end{array}$} & \multicolumn{3}{|c|}{ Peak coordinates } & \multirow{2}{*}{$\begin{array}{l}\text { Peak } \\
t\end{array}$} \\
\hline & & & & $x$ & $\mathrm{Y}$ & Z & \\
\hline \multicolumn{8}{|c|}{ Unusual versus ordinary } \\
\hline \multirow[t]{5}{*}{ TD } & Inferior parietal lobule & Left & 2,110 & -45 & -45 & 42 & 9.0 \\
\hline & Superior occipital gyrus & Right & 1,239 & 30 & -75 & 18 & 7.0 \\
\hline & Precentral gyrus & Left & 1,015 & -45 & 3 & 18 & 5.7 \\
\hline & Angular gyrus & Right & 385 & 27 & -63 & 45 & 7.3 \\
\hline & Caudate & Left & 284 & -9 & 9 & 6 & 4.5 \\
\hline \multirow[t]{4}{*}{ ASD } & Inferior temporal gyrus & Left & 2,608 & -45 & -60 & -6 & 6.7 \\
\hline & Postcentral gyrus & Left & 2,525 & -51 & -3 & 39 & 6.3 \\
\hline & Middle occipital gyrus & Right & 1,912 & 36 & -75 & 12 & 5.9 \\
\hline & Inferior frontal gyrus & Right & 801 & 33 & 30 & -6 & 5.3 \\
\hline \multicolumn{8}{|c|}{ Ordinary versus unusual } \\
\hline \multirow[t]{8}{*}{ TD } & Calcarine gyrus & Right & 1,416 & 3 & -60 & 12 & -6.2 \\
\hline & Postcentral gyrus & Left & 391 & -45 & -21 & 48 & -5.2 \\
\hline & Middle temporal gyrus & Left & 303 & -45 & -39 & 0 & -5.1 \\
\hline & Angular gyrus & Right & 216 & 45 & -69 & 36 & -4.1 \\
\hline & Superior frontal gyrus & Right & 197 & 24 & 63 & 9 & -4.0 \\
\hline & Middle frontal gyrus & Right & 168 & 27 & 24 & 36 & -5.7 \\
\hline & Superior temporal gyrus & Right & 159 & 39 & -27 & 0 & -4.6 \\
\hline & Middle frontal gyrus & Left & 137 & -30 & 30 & 33 & -4.0 \\
\hline \multirow[t]{3}{*}{ ASD } & Calcarine gyrus & Right & 1,855 & 12 & -78 & 15 & -5.3 \\
\hline & Thalamus & Right & 405 & 6 & -27 & 12 & -4.6 \\
\hline & Postcentral gyrus & Right & 175 & 39 & -27 & 51 & -5.2 \\
\hline \multicolumn{8}{|l|}{ Group differences } \\
\hline \multirow[t]{4}{*}{$A S D>T D$} & Middle orbital gyrus & Right & 273 & 48 & 54 & -9 & -4.0 \\
\hline & Precentral gyrus & Right & 258 & 51 & 9 & 42 & -4.9 \\
\hline & Postcentral gyrus & Left & 246 & -39 & -30 & 60 & -4.5 \\
\hline & Inferior parietal lobule & Right & 154 & 45 & -48 & 54 & -4.2 \\
\hline
\end{tabular}

Abbreviations: TD typically developing, ASD autism spectrum disorder.

participants with ASD showed increased recruitment of areas involved in action understanding; IFG and IPL for processing intention and unusual actions respectively.

\section{Functional connectivity}

When connectivity among pairs of ROIs were examined, the TD group showed increased functional connectivity $(r=0.64)$ compared to the ASD group $(r=0.53)$ between RSTS/RTPJ and right superior/middle frontal gyrus in the intention condition $(t(41)=2.12, P=0.04$, uncorrected). However, this effect did not survive multiple comparisons correction. Nevertheless, a follow-up connectivity network analysis revealed a statistically significant difference (corrected using the Holm-Bonferroni method) between groups, with the ASD group showing reduced frontaltemporal functional connectivity, relative to TD controls, during intention $(t(41)=2.90, P=0.006$, corrected) (see Figure 4). No significant group differences in connectivity were detected while processing the means of actions.

\section{Discussion}

By asking participants to judge the nature of intentions and means (unusual or ordinary) of a model's actions, this study targeted two important neural networks mediating action understanding, the MNS and the ToM, in autism. It has been a topic of debate in the field of social neuroscience as to whether these two networks are mutually exclusive $[80,81]$ or functionally coordinated $[2,82,83]$ in the context of processing action intentions. In our study, both groups showed increased activation in middle/superior temporal cortex and PCC/precuneus, while processing intentions of actions relative to processing means of actions; regions that are considered part of the ToM network. Processing means, 


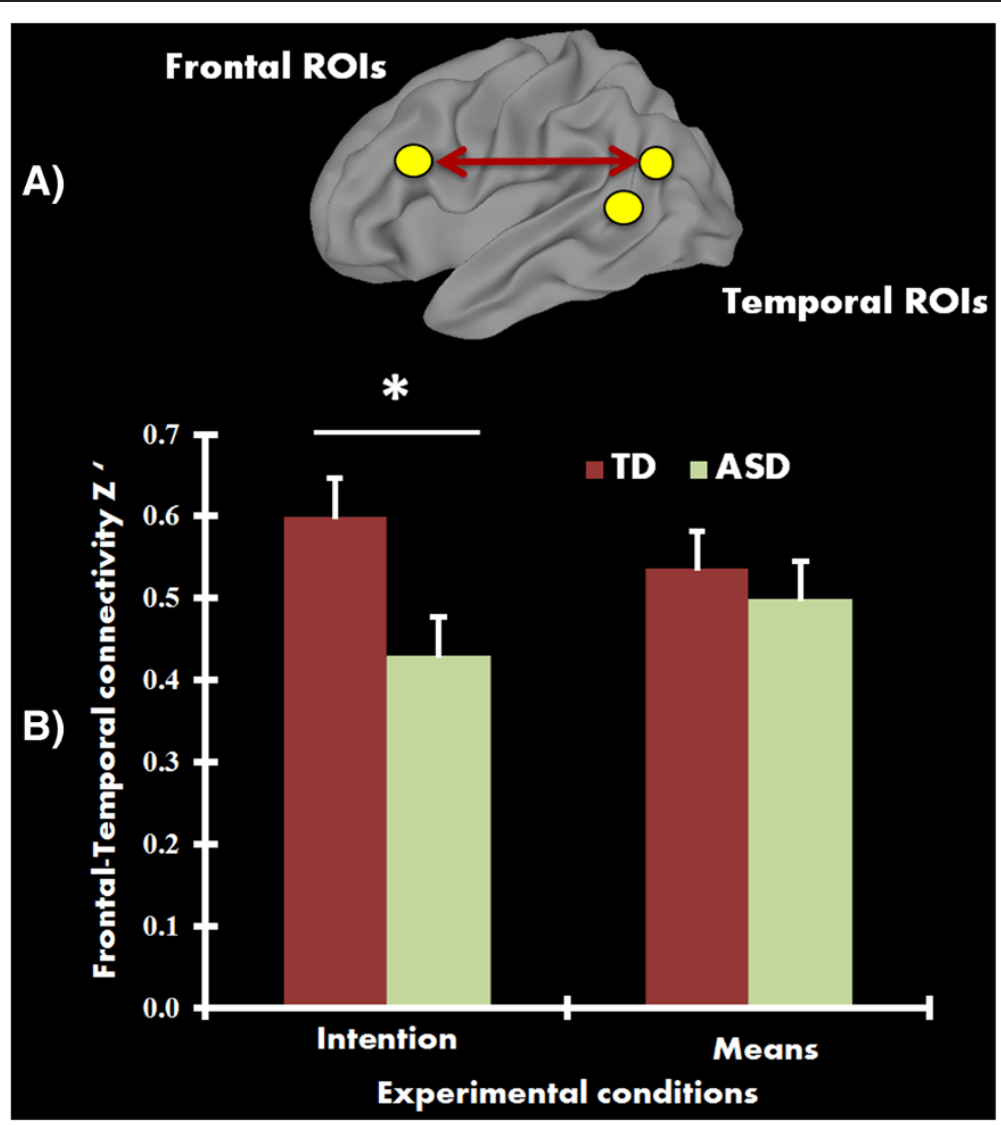

Figure 4 Functional connectivity results. A) Surface rendering showing frontal-temporal connections (regions of interest (ROIs) do not represent actual size) that revealed reduced functional connectivity in the autism spectrum disorder (ASD) group. B) Mean functional connectivity $\left(Z^{\prime}\right)$ in the frontal-temporal network for each group for intention and means conditions. Participants with ASD showed significantly reduced functional connectivity $(P<0.01$, corrected) relative to typically developing (TD) control participants in the intention condition, but not in the means condition. Error bars represent standard error of the mean.

on the other hand, showed greater activity in inferior parietal (part of the MNS network) and occipital areas. Neurons in the IPL have been found to be critical in coding the organization of motor acts and in understanding observed actions $[84,85]$. The pattern of activation seen within each group in our study suggests the use of mentalizing and mirroring mechanisms in inferring intentions and means of actions respectively. Nevertheless, it should be noted that processing intentions, relative to means, also elicited more activity in the frontal areas (MFG and IFG) in the ASD group.

This pattern was also seen in the between-group analysis, where the ASD group showed significantly more activity, relative to TD, in left IFG for processing intention. On the other hand, the ASD group showed reduced activity, for the same contrast, in the right calcarine sulcus extending to PCC. Thus, the ASD participants showed reduced activity in a core ToM network area and increased activity in a core MNS area, albeit in the left hemisphere. The left IFG peak activation was found in Brodmann area (BA) 45 and it is possible that the ASD participants depicted action intentions as pragmatic representations (the parameters that are relevant to the motor commands when executing and observing an action), which were strongly associated with the visual cue [86]. A recent meta-analysis of studies of action understanding found that bilateral BA44 and BA45 differed in activity, with the former more involved action imitation and the latter in action observation [87]. While IFG activation is attributed to MNS usually in the right hemisphere, there are several studies showing the role of LIFG in action understanding. For example, using a repetition suppression paradigm, Pobric and Hamilton showed that LIFG is necessary in making perceptual judgment about people's actions [88]. BA45, especially in the left hemisphere, has also been associated with language production, and this increased activation may underlie communication disorders experienced by people with autism [89]. Another possible explanation is that our stimuli for identifying intentions may have targeted motor intentions, rather than abstract intentions, and ASD individuals may have processed this at the motor level with increased recruitment of the left IFG. Therefore, 
it is possible that ASD participants, while inferring the intent behind actions, might have relied on perceptual, motoric, and linguistic representations of actions more, as evidenced by the increased LIFG activation. It should also be noted that the TD participants in our study activated BA47, which encodes more abstract semantic representations of an action; however, this cluster did not survive multiple comparisons correction. The ASD participants did not activate this region using the same uncorrected threshold.

Most of the findings in this study are consistent with that of de Lange et al. [18], from where the current stimuli are adapted. Specifically, the TD group's activation in PCC and middle temporal gyrus extending to bilateral TPJ while attending to intentions, as well as activation in bilateral occipital areas while attending to means, are consistent with this study. However, unlike the de Lange study we did not find MPFC activation at the same corrected statistical threshold at which we report other results. The MPFC has been linked primarily to self-other reflections. In the current study, the participants may have refrained from self-reflection or abstract thinking as the intentions of the model were common and evident at the motor level [90-93]. In our study, the inference about intentions may emerge from determining the model's goal when holding a spoon (to eat) or a cup (to drink). Whereas the original study by de Lange et al. included images where both the how and why of an action could be unusual, our study only included unusual trials where one aspect of the action was unusual and the other was ordinary, thus making the current task relatively easier for participants. This could be another reason for the absence of MPFC activation in our task.

Previous studies have reported that prior experience, visual or motor, with an action can help in predicting the means and intentions behind that action [94-96]. Thus, the cortical mechanisms mediating a familiar action may be different from that of an unfamiliar action. In the current study, we also tested how MNS and ToM networks responded to unusual and ordinary actions and how it differed in ASD individuals. Judging unusual actions, when compared to ordinary actions, showed activation primarily in the MNS (bilateral IPL, SMA, and IFG) along with occipital regions extending to EBA in both groups. These findings are consistent with previous studies that have also reported activation in some of these regions when viewing irrational actions versus rational actions [97,98]. In addition, occipital activation during unusual actions correspond to other studies where strong EBA response has been previously reported during incoherent actions [99]. The increased left IFG activation in the ASD group while judging means, and increased activity in the ventral premotor and IPL during unusual actions in the current study is consistent with a previous report of such an increase [46], where participants also activated MNS network during a particular task.

Reduced functional connectivity of the frontal/posterior temporal network in the ASD group while judging intentions may suggest a more isolated and less coordinated way of processing information in individuals with ASD. This weaker connectivity in ASD is in line with previous studies in adults with ASD reporting significant disruptions in frontal to posterior connectivity [58,60-62]. The TD control participants here may be effectively engaging and coordinating frontal and temporal regions in processing actions at multiple levels (perceptual, motor, goal-oriented or intentional), ultimately inferring the intention behind actions. Such communication may not be the norm in information processing in ASD participants. While STS/TPJ and IFG belong to seemingly different, anatomically segregated networks (ToM and MNS respectively), their functional communication may be important in understanding actions at richer and more comprehensive levels. While ASD participants understand the intentions behind these actions more accurately, it is possible that their neural and cognitive route may differ from TD control participants, and may not be processing intentions at the same level as the TD control participants. It should also be noted that a group difference in connectivity was absent while processing the means of actions $(r=0.57$ and 0.49 for TD and ASD, respectively). Efficient crosstalk between frontal and temporal regions in TD participants may underlie flexible modulation of functions (mirroring and mentalizing), which may be absent in ASD. Thus, the atypical connectivity between these two networks may be modulated by the different tasks in our study: intention, which primarily targeted ToM activity, and means, which primarily targeted MNS activity. Such a pattern has also been observed in resting-state fcMRI studies, where intrinsic BOLD signal fluctuations (as opposed to the task-driven effects in our study) are assessed [100]. Examining effective connectivity may provide more information about the transfer of information between these networks and provide more insights into the functional dynamics of this communication.

An exploratory correlational analysis revealed two significant relationships between PCC activation (intention versus means) with RAADS-R and EQ scores for all participants (ASD and TD groups combined). While these two relationships are significant, their effect size may not reveal a strong correlational effect. Nevertheless, these results are meaningful in that the activation in PCC decreased with an increase in symptom severity, and the activation in PCC increased with the ability to empathize. Finally, at the behavioral level, although a significant difference in accuracy for processing means and intentions was found when comparing the ASD young adults to their TD peers, all participants performed well above chance on the task, 
with overall group means for accuracy appearing to be very high (over $80 \%$ for each condition and group).

\section{Conclusions}

In summary, we found differential activation in the TD group while processing intention and means, whereas this effect was less prominent in the ASD group. The participants with ASD showed robust MNS activity in IFG and IPL while coding intention and means of this action-understanding task. Some of these regions showed significantly increased activity in ASD participants when compared to their TD peers. Despite significant activation, ASD participants showed weaker connectivity, relative to TD controls, between frontal and temporoparietal areas while processing intention. Overall, these findings suggest a complex pattern of MNS and ToM response in individuals with ASD, with mostly intact MNS response accompanied by altered ToM activation and connectivity. Future studies should further examine the anatomical and functional roles of these systems in different aspects of action understanding and social cognition in autism.

\begin{abstract}
Abbreviations
ANOVA: Analyses of variance; ASD: Autism spectrum disorder; BOLD: Bloodoxygen-level dependent; EBA: Extrastriate body area; EQ: Empathy Quotient; fCMRI: Functional connectivity MRI; fMRI: Functional magnetic resonance imaging; IFG: Inferior frontal gyrus; IPL: Inferior parietal lobule; IQ: Intelligence quotient; MCIN: Middle cingulate; MFG: Middle frontal gyrus; MNS: Mirror neuron system; MPFC: Medial prefrontal cortex; PCC: Posterior cingulate cortex; RAADS-R: Ritvo Autism Asperger Diagnostic Scale-Revised; ROI: Region of interest; RT: Reaction time; STS: Superior temporal sulcus; TD: Typically developing; ToM: Theory of mind; TPJ: Temporoparietal junction; UAB: University of Alabama at Birmingham; WASI: Wechsler Abbreviated Scale of Intelligence.
\end{abstract}

\section{Competing interests}

The authors declare that they have no competing interests.

\section{Authors' contributions}

LEL participated in the design of the study, collected the data, performed the statistical analyses and drafted the manuscript. JOM performed the statistical analyses and drafted the manuscript. HDD assisted with protocol design, data collection, statistical analyses, computational support, and manuscript preparation. LGK and MRK helped in protocol design, participant characterization, recruitment, and in manuscript preparation. RKK conceived the study, participated in the design of the study, and drafted the manuscript. All authors read and approved the final manuscript.

\section{Acknowledgments}

The authors would like to thank Dr Marie Moore Channell, and Patrick Powell for their help at different stages of this study. We would also like to thank Dr Floris de Lange for generously providing the stimuli for this study. This study was supported by the UAB Department of Psychology Faculty funds and the McNulty-Civitan Scientist Award to RK.

\section{Author details}

'Department of Psychology, University of Alabama at Birmingham, CIRC 235G, 1719 6th Ave South, Birmingham, AL 35294-0021, USA. ²Department of Radiology, University of Alabama at Birmingham, CIRC 235F, 1719 6th Ave South, Birmingham, AL 35294-0021, USA. ${ }^{3}$ Treatment and Education of Autistic and Communication related handicapped CHildren (TEACCH) Autism Program, University of North Carolina School of Medicine, Campus Box 7180, UNC-Chapel Hill, Chapel Hill, NC 27510, USA. ${ }^{4}$ Department of Allied Health Sciences, University of North Carolina, Bondurant Hall, CB \#7120, Chapel Hill, NC 25799-7120, USA
Received: 4 June 2014 Accepted: 26 September 2014

Published: 14 October 2014

\section{References}

1. Thioux M, Gazzola V, Keysers C: Action understanding: how, what and why. Curr Biol 2008, 18:R431-R434.

2. Gallese V, Goldman A: Mirror neurons and the simulation theory of mind-reading. Trends Cogn Sci 1998, 2:493-501.

3. Gordon RM: Folk psychology as simulation. Mind Lang 1986, 1:158-171.

4. Heal J: Replication and functionalism. New York, NY: Cambridge University Press; 1986.

5. Avikainen S, Forss N, Hari R: Modulated activation of the human SI and SII cortices during observation of hand actions. Neuroimage 2002, 15:640-646.

6. Gazzola V, Aziz-Zadeh L, Keysers C: Empathy and the somatotopic auditory mirror system in humans. Curr Biol 2006, 16:1824-1829.

7. Gazzola V, Rizzolatti G, Wicker B, Keysers C: The anthropomorphic brain: the mirror neuron system responds to human and robotic actions. Neuroimage 2007, 35:1674-1684.

8. Gallese V, Fadiga L, Fogassi L, Rizzolatti G: Action recognition in the premotor cortex. Brain 1996, 119:593-609.

9. Gallese V, Keysers C, Rizzolatti G: A unifying view of the basis of social cognition. Trends Cogn Sci 2004, 8:396-403.

10. Keysers C, Kohler E, Umiltà MA, Nanetti L, Fogassi L, Gallese V: Audiovisual mirror neurons and action recognition. Exp Brain Res 2003, 153:628-636.

11. Kohler E, Keysers C, Umilta MA, Fogassi L, Gallese V, Rizzolatti G: Hearing sounds, understanding actions: action representation in mirror neurons. Science 2002, 297:846-848.

12. Rizzolatti G, Fadiga L, Matelli M, Bettinardi V, Paulesu E, Perani D, Fazio F: Localization of grasp representations in humans by PET: 1. Observation versus execution. Exp Brain Res 1996, 111:246-252.

13. Grafton ST, Hamilton AF d C: Evidence for a distributed hierarchy of action representation in the brain. Hum Mov Sci 2007, 26:590-616.

14. Hari R, Forss N, Avikainen S, Kirveskari E, Salenius S, Rizzolatti G: Activation of human primary motor cortex during action observation: a neuromagnetic study. Proc Natl Acad Sci 1998, 95:15061-15065.

15. Rizzolatti G, Fadiga L: Grasping objects and grasping action meanings: the dual role of monkey rostroventral premotor cortex (area F5). Novartis Found Symp 1998, 218:81-95. discussion 95-103.

16. Keysers C, Gazzola V: Integrating simulation and theory of mind: from self to social cognition. Trends Cogn Sci 2007, 11:194-196.

17. Uddin LQ, lacoboni M, Lange C, Keenan JP: The self and social cognition: the role of cortical midline structures and mirror neurons. Trends Cogn SCi 2007, 11:153-157.

18. de Lange FP, Spronk M, Willems RM, Toni I, Bekkering H: Complementary systems for understanding action intentions. CB 2008, 18:454-457.

19. Fletcher PC, Happe F, Frith U, Baker SC, Dolan RJ, Frackowiak RS, Frith CD: Other minds in the brain: a functional imaging study of "theory of mind" in story comprehension. Cognition 1995, 57:109-128.

20. Gallagher HL, Happe F, Brunswick N, Fletcher PC, Frith U, Frith CD: Reading the mind in cartoons and stories: an fMRI study of "theory of mind" in verbal and nonverbal tasks. Neuropsychologia 2000, 38:11-21.

21. Ruby P, Decety J: What you believe versus what you think they believe: a neuroimaging study of conceptual perspective taking. Eur J Neurosci 2003, 17:2475-2480.

22. Saxe R, Kanwisher N: People thinking about thinking people: the role of the temporo-parietal junction in "theory of mind". Neuroimage 2003, 19:1835-1842.

23. Vogeley K, Bussfeld P, Newen A, Herrmann S, Happe F, Falkai P, Maier W, Shah N, Fink G, Zilles K: Mind reading: neural mechanisms of theory of mind and self-perspective. Neuroimage 2001, 14:170-181.

24. Van Overwalle F, Baetens K: Understanding others' actions and goals by mirror and mentalizing systems: a meta-analysis. Neuroimage 2009, 48:564-584.

25. Spunt RP, Falk EB, Lieberman MD: Dissociable neural systems support retrieval of how and why action knowledge. Psychol Sci 2010, 21:1593-1598.

26. Baron-Cohen S, Leslie AM, Frith U: Does the autistic child have a "theory of mind"? Cognition 1985, 21:37-46.

27. Baron-Cohen S: Mindblindness: An essay on autism and theory of mind. Cambridge, MA: Bradford: MIT Press; 1995. 
28. Happé F, Frith U: The neuropsychology of autism. Brain 1996, 119:1377-1400.

29. Williams JH, Waiter GD, Gilchrist A, Perrett DI, Murray AD, Whiten A: Neural mechanisms of imitation and 'mirror neuron'functioning in autistic spectrum disorder. Neuropsychologia 2006, 44:610-621.

30. Meltzoff AN, Gopnik A: The role of imitation in understanding persons and developing a theory of mind. In Understanding other minds: Perspectives from autism. Edited by Baron-Cohen S, Tager-Flusburg E. Cohen, D. New York, NY: Oxford University Press; 1993:335-366.

31. Rogers SJ, Pennington BF: A theoretical approach to the deficits in infantile autism. Dev Psychopathol 1991, 3:137-162.

32. Rogers SJ: An examination of the imitation deficit in autism. New York, NY: Cambridge University Press; 1999

33. Smith IM, Bryson SE: Imitation and action in autism: a critical review. Psychol Bull 1994, 116:259.

34. Heiser M, lacoboni M, Maeda F, Marcus J, Mazziotta JC: The essential role of Broca's area in imitation. Eur J Neurosci 2003, 17:1123-1128.

35. lacoboni M, Woods RP, Brass M, Bekkering H, Mazziotta JC, Rizzolatti G: Cortical mechanisms of human imitation. Science 1999, 286:2526-2528.

36. lacoboni M, Dapretto M: The mirror neuron system and the consequences of its dysfunction. Nat Rev Neurosci 2006, 7:942-951.

37. Koski L, Wohlschläger A, Bekkering H, Woods RP, Dubeau M-C, Mazziotta JC, lacoboni M: Modulation of motor and premotor activity during imitation of target-directed actions. Cereb Cortex 2002, 12:847-855.

38. Koski L, lacoboni M, Dubeau M-C, Woods RP, Mazziotta JC: Modulation of cortical activity during different imitative behaviors. J Neurophysio/ 2003, 89:460-471.

39. Oberman LM, Ramachandran VS: The simulating social mind: the role of the mirror neuron system and simulation in the social and communicative deficits of autism spectrum disorders. Psychol Bull 2007, 133:310.

40. Oberman LM, Hubbard EM, McCleery JP, Altschuler EL, Ramachandran VS, Pineda JA: EEG evidence for mirror neuron dysfunction in autism spectrum disorders. Cogn Brain Res 2005, 24:190-198.

41. Kana RK, Libero LE, Hu CP, Deshpande HD, Colburn JS: Functional brain networks and white matter underlying theory-of-mind in autism. Soc Cogn Affect Neurosci 2014, 9:98-105.

42. Cattaneo L, Fabbri-Destro M, Boria S, Pieraccini C, Monti A, Cossu G, Rizzolatti G: Impairment of actions chains in autism and its possible role in intention understanding. Proc Natl Acad Sci 2007, 104:17825-17830.

43. Dapretto M, Davies MS, Pfeifer JH, Scott AA, Sigman M, Bookheimer SY, lacoboni M: Understanding emotions in others: mirror neuron dysfunction in children with autism spectrum disorders. Nat Neurosci 2005, 9:28-30

44. Hadjikhani N, Joseph RM, Snyder J, Tager-Flusberg H: Anatomical differences in the mirror neuron system and social cognition network in autism. Cereb Cortex 2006, 16:1276.

45. Gallese V: Intentional attunement: a neurophysiological perspective on social cognition and its disruption in autism. Brain Res 2006, 1079:15-24.

46. Martineau J, Andersson F, Barthélémy C, Cottier J-P, Destrieux C: Atypical activation of the mirror neuron system during perception of hand motion in autism. Brain Res 2010, 1320:168-175.

47. Hamilton AFC: Reflecting on the mirror neuron system in autism: a systematic review of current theories. Dev Cogn Neurosci 2013, 3:91-105.

48. Bernier R, Dawson G: The role of mirror neuron dysfunction in autism. In Mirror Neuron Systems. Heidelberg: Springer; 2009:261-286.

49. Kana RK, Wadsworth HM, Travers BG: A systems level analysis of the mirror neuron hypothesis and imitation impairments in autism spectrum disorders. Neurosci Biobehav Rev 2011, 35:894-902.

50. Williams JH: Self-other relations in social development and autism: multiple roles for mirror neurons and other brain bases. Autism Res 2008, 1:73-90.

51. Avikainen S, Kulomäki T, Hari R: Normal movement reading in Asperger subjects. Neuroreport 1999, 10:3467-3470.

52. Dinstein I, Thomas C, Behrmann M, Heeger DJ: A mirror up to nature. Curr Biol 2008, 18:R13-R18.

53. Raymaekers R, Wiersema JR, Roeyers H: EEG study of the mirror neuron system in children with high functioning autism. Brain Res 2009, 1304:113-121.

54. Hamilton AFC, Brindley RM, Frith U: Imitation and action understanding in autistic spectrum disorders: how valid is the hypothesis of a deficit in the mirror neuron system? Neuropsychologia 2007, 45:1859-1868.

55. Southgate $V$, Hamilton AFDC: Unbroken mirrors: challenging a theory of autism. Trends Cogn Sci 2008, 12:225-229.
56. Dinstein I, Thomas C, Humphreys K, Minshew N, Behrmann M, Heeger DJ: Normal movement selectivity in autism. Neuron 2010, 66:461-469.

57. Castelli F, Frith C, Happé F, Frith U: Autism, Asperger syndrome and brain mechanisms for the attribution of mental states to animated shapes. Brain 2002, 125:1839-1849.

58. Kana RK, Keller TA, Cherkassky VL, Minshew NJ, Just MA: Atypical frontal-posterior synchronization of Theory of Mind regions in autism during mental state attribution. Soc Neurosci 2009, 4:135-152.

59. Pelphrey K, Adolphs R, Morris JP: Neuroanatomical substrates of social cognition dysfunction in autism. Ment Retard Dev Disabil Res Rev 2004, 10:259-271.

60. Kana RK, Libero LE, Moore MS: Disrupted cortical connectivity theory as an explanatory model for autism spectrum disorders. Phys Life Rev 2011, 8:410-437.

61. Just MA, Keller TA, Malave VL, Kana RK, Varma S: Autism as a neural systems disorder: a theory of frontal-posterior underconnectivity. Neurosci Biobehav Rev 2012, 36:1292-1313.

62. Schipul SE, Keller TA, Just MA: Inter-regional brain communication and its disturbance in autism. Front Syst Neurosci 2011, 5:7.

63. Maximo JO, Cadena EJ, Kana RK: The implications of brain connectivity in the neuropsychology of autism. Neuropsychol Rev 2014, 24:16-31.

64. Wechsler D: Wechsler abbreviated scale of intelligence. San Antonio, TX: Psychological Corporation; 1999.

65. Oldfield RC: The assessment and analysis of handedness: the Edinburgh inventory. Neuropsychologia 1971, 9:97-113.

66. Baron-Cohen S, Wheelwright S, Hill J, Raste Y, Plumb I: The "Reading the Mind in the Eyes" Test revised version: a study with normal adults, and adults with Asperger syndrome or high-functioning autism. J Child Psychol Psychiatry 2001, 42:241-251.

67. Ritvo RA, Ritvo ER, Guthrie D, Ritvo MJ, Hufnagel DH, McMahon W, Tonge B, Mataix-Cols D, Jassi A, Attwood T: The Ritvo Autism asperger diagnostic scale-revised (RAADS-R): a scale to assist the diagnosis of Autism spectrum disorder in adults: an international validation study. J Autism Dev Disord 2011, 41:1076-1089.

68. Baron-Cohen S, Wheelwright S: The empathy quotient: an investigation of adults with Asperger syndrome or high functioning autism, and normal sex differences. J Autism Dev Disord 2004, 34:163-175.

69. Lord C, Rutter M, Le Couteur A: Autism Diagnostic Interview-Revised: a revised version of a diagnostic interview for caregivers of individuals with possible pervasive developmental disorders. J Autism Dev Disord 1994, 24:659-685.

70. Lord C, Risi S, Lambrecht L, Cook EH Jr, Leventhal BL, DiLavore PC, Pickles A, Rutter M: The autism diagnostic observation schedule-generic: a standard measure of social and communication deficits associated with the spectrum of autism. J Autism Dev Disord 2000, 30:205-223.

71. Cox RW: AFNI: software for analysis and visualization of functional magnetic resonance neuroimages. Comput Biomed Res 1996, 29:162-173.

72. Smith SM, Jenkinson M, Woolrich MW, Beckmann CF, Behrens TE, Johansen-Berg H, Bannister PR, De Luca M, Drobnjak I, Flitney DE, Niazy RK, Saunders J, Vickers J, Zhang Y, De Stefano N, Brady JM, Matthews PM: Advances in functional and structural MR image analysis and implementation as FSL. Neuroimage 2004, 23(Suppl 1):S208-S219.

73. Jenkinson M, Bannister P, Brady M, Smith S: Improved optimization for the robust and accurate linear registration and motion correction of brain images. Neuroimage 2002, 17:825-841.

74. Jenkinson M, Smith S: A global optimisation method for robust affine registration of brain images. Med Image Anal 2001, 5:143-156.

75. Zhang $Y$, Brady M, Smith S: Segmentation of brain MR images through a hidden Markov random field model and the expectation-maximization algorithm. IEEE Trans Med Imaging 2001, 20:45-57.

76. Nair A, Keown CL, Datko M, Shih P, Keehn B, Müller RA: Impact of methodological variables on functional connectivity findings in Autism Spectrum Disorders. Hum Brain Mapp 2014, 35:4035-4048.

77. Van Dijk KR, Sabuncu MR, Buckner RL: The influence of head motion on intrinsic functional connectivity MRI. Neuroimage 2012, 59:431-438

78. Satterthwaite TD, Elliott MA, Gerraty RT, Ruparel K, Loughead J, Calkins ME, Eickhoff SB, Hakonarson H, Gur RC, Gur RE: An improved framework for confound regression and filtering for control of motion artifact in the preprocessing of resting-state functional connectivity data. Neuroimage 2013, 64:240-256. 
79. Power JD, Barnes KA, Snyder AZ, Schlaggar BL, Petersen SE: Spurious but systematic correlations in functional connectivity MRI networks arise from subject motion. Neuroimage 2012, 59:2142-2154.

80. Saxe $R$, Wexler A: Making sense of another mind: the role of the right temporo-parietal junction. Neuropsychologia 2005, 43:1391-1399.

81. Southgate V, Johnson MH, El Karoui I, Csibra G: Motor system activation reveals infants' on-line prediction of others' goals. Psychol Sci 2010, 21:355-359.

82. Rizzolatti G, Fadiga L, Fogassi L, Gallese V: Resonance behaviors and mirror neurons. Arch Ital Biol 1999, 137:85-100.

83. Rizzolatti $\mathrm{G}$, Sinigaglia $\mathrm{C}$ : The functional role of the parieto-frontal mirror circuit: interpretations and misinterpretations. Nat Rev Neurosci 2010, 11:264-274.

84. Fogassi L, Ferrari PF, Gesierich B, Rozzi S, Chersi F, Rizzolatti G: Parietal lobe: from action organization to intention understanding. Science 2005, 308:662-667.

85. Rizzolatti G, Fogassi L, Gallese V: Mirrors of the mind. Sci Am 2006, 295:54-61

86. Kilner JM: More than one pathway to action understanding. Trends Cogn Sci 2011, 15:352-357.

87. Caspers S, Zilles K, Laird AR, Eickhoff SB: ALE meta-analysis of action observation and imitation in the human brain. Neuroimage 2010, 50:1148-1167.

88. Pobric $\mathrm{G}$, Hamilton $\mathrm{A}$ : Action understanding requires the left inferior frontal gyrus. Curr Biol 2006, 5:524-529.

89. Kleinhans NM, Muller RA, Cohen DN, Courchesne E: Atypical functional lateralization of language in autism spectrum disorders. Brain Res 2008, 1221:115-125.

90. Mitchell JP, Banaji MR, MacRae CN: The link between social cognition and self-referential thought in the medial prefrontal cortex. J Cogn Neurosci 2005, 17:1306-1315.

91. Gusnard DA, Akbudak E, Shulman GL, Raichle ME: Medial prefrontal cortex and self-referential mental activity: relation to a default mode of brain function. Proc Natl Acad Sci 2001, 98:4259-4264.

92. D'Argembeau A, Ruby P, Collette F, Degueldre C, Balteau E, Luxen A, Maquet $P$, Salmon E: Distinct regions of the medial prefrontal cortex are associated with self-referential processing and perspective taking. J Cogn Neurosci 2007, 19:935-944.

93. Heatherton TF, Wyland CL, Macrae CN, Demos KE, Denny BT, Kelley WM: Medial prefrontal activity differentiates self from close others. Soc Cogn Affect Neurosci 2006, 1:18-25.

94. Knoblich G, Flach R: Predicting the effects of actions: interactions of perception and action. Psychol Sci 2001, 12:467-472.

95. Graf M, Reitzner B, Corves C, Casile A, Giese M, Prinz W: Predicting point-light actions in real-time. Neuroimage 2007, 36:T22-T32.

96. Springer A, Brandstädter S, Liepelt R, Birngruber T, Giese M, Mechsner F, Prinz W: Motor execution affects action prediction. Brain Cogn 2011, 76:26-36.

97. Marsh LE, Hamilton AFC: Dissociation of mirroring and mentalising systems in autism. Neuroimage 2011, 56:1511-1519.

98. Cross ES, Mackie EC, Wolford G, Hamilton AFC: Contorted and ordinary body postures in the human brain. Exp Brain Res 2010, 204:397-407.

99. Downing PE, Peelen MV, Wiggett AJ, Tew BD: The role of the extrastriate body area in action perception. Soc Neurosci 2006, 1:52-62.

100. Fishman I, Keown CL, Lincoln AJ, Pineda JA, Müller R-A: Atypical cross talk between mentalizing and mirror neuron networks in autism spectrum disorder. JAMA psychiatry 2014, 71:751-760.

\section{Submit your next manuscript to BioMed Central and take full advantage of:}

- Convenient online submission

- Thorough peer review

- No space constraints or color figure charges

- Immediate publication on acceptance

- Inclusion in PubMed, CAS, Scopus and Google Scholar

- Research which is freely available for redistribution

Submit your manuscript at www.biomedcentral.com/submit
C Biomed Central 